\title{
The Influence of Gamma Irradiation on Space Charge in LDPE
}

\author{
$\mathrm{G} \mathrm{Chen}^{1 *}$ and $\mathrm{M} \mathrm{Fu}^{2}$ \\ ${ }^{1}$ School of Electronics and Computer Science \\ University of Southampton UK \\ ${ }^{2}$ Department of Engineering \\ University of Leicester UK \\ *Email: gc@ecs.soton.ac.uk
}

\begin{abstract}
In the present paper, we report an investigation on the effect of radiation environment on space charge in low density polyethylene (LDPE). LDPE thin films of $\sim 100 \mu \mathrm{m}$ were irradiated at a dose rate of approximately $1 \mathrm{kGy} / \mathrm{h}$ using a ${ }^{60} \mathrm{Co}$ gamma source. The samples were irradiated in air, vacuum and nitrogen gas at room temperature to various doses from 5 to $100 \mathrm{kGy}$. Space charge measurements were carried out using the pulsed electroacoustic (PEA) technique. The applied electric field in all cases is $50 \mathrm{kV} / \mathrm{mm}$. It has been found that the space charge dynamics in the irradiated samples are different from the fresh (un-irradiated) sample and affected by irradiation environment. Although the oxidation of the samples in air may play an important role in the observed differences, charge dynamics in the samples irradiated in vacuum and pure nitrogen gas are also different.
\end{abstract}

\section{INTRODUCTION}

Ionising radiation can profoundly alter the molecular structure and thereby the macroscopic properties of polymeric materials through mechanisms like chain scission, cross-linking and oxidation. Radiation effects in polymers have been studied for many years to investigate various aspects of material behaviour [1-4] and among those aspects has featured the electrical response to radiation. It has been suggested that radiation may give rise to the presence of trapped charge within the material, the trapping characteristics of which may be influenced by these radiation-induced structural alterations. Earlier work related particularly to the effects of radiation on the electrical behaviour of low-density polyethylene (LDPE) has shown that a radiation environment can influence the thermally stimulated discharge current (TSDC) spectra in the high temperature region, with more charge being found within the sample irradiated in air compared to those irradiated in nitrogen [5]; the dc electrical conductivity of LDPE irradiated in air is significantly greater than of material irradiated in nitrogen [6]; and the pre-breakdown transient current pulse activity in LDPE under direct stress has been demonstrated to be much greater in material irradiated in air compared to that irradiated in nitrogen [7]. It has generally been considered that space charge played a role in the above behaviour of irradiated materials.
In the present paper, we report an investigation on the effect of radiation environment on space charge in low density polyethylene (LDPE). LDPE thin films of $\sim 100$ $\mu \mathrm{m}$ were irradiated at a dose rate of approximately 1 $\mathrm{kGy} / \mathrm{h}$ using a ${ }^{60} \mathrm{Co}$ gamma source. The samples were irradiated in air, vacuum and nitrogen gas at room temperature to various doses from 5 to $100 \mathrm{kGy}$. Space charge measurements were carried out using the pulsed electroacoustic (PEA) technique.

\section{EXPERIMENTAL METHOD}

\section{Sample Preparation}

To minimise the morphological differences and charge trapping that may arise from the presence of additives, $100 \mu \mathrm{m}$ additive free LDPE film was used in the present study. LDPE samples were cut from the film, cleaned with alcohol and subsequently placed in a ${ }^{60} \mathrm{Co}$ gamma source for irradiation to different doses between $5 \mathrm{kGy}$ and 100 $\mathrm{kGy}$ at a dose rate of approximately $1 \mathrm{kGy} / \mathrm{h}$. Different batches of samples were irradiated at room temperature in ambient air, oxygen-free nitrogen and vacuum. For irradiation in nitrogen and vacuum, the samples were placed inside glass tubes which were evacuated for several hours before being sealed (vacuum) or backfilled with nitrogen gas and sealed.

\section{Measurements}

Measurements of space charge were carried out using the pulsed electroacoustic technique (PEA) [8,9]. Test specimens were stressed electrically under dc at 50 $\mathrm{kV} / \mathrm{mm}$ for 60 minutes. Charge distributions were recorded at various times and charge decay after the removal of the applied stress was also monitored. All the measurements were made at room temperature.

\section{RESULTS AND DISCUSSION}

\section{Control Sample}

To observe the influence of irradiation on space charge formation in LDPE, reference space charge distributions were obtained with unirradiated control samples at 50 $\mathrm{kV} / \mathrm{mm}$. The charge distribution in the control sample 
following stressing for 60 minutes at $50 \mathrm{kV} / \mathrm{mm}$ is displayed in Figure 1.

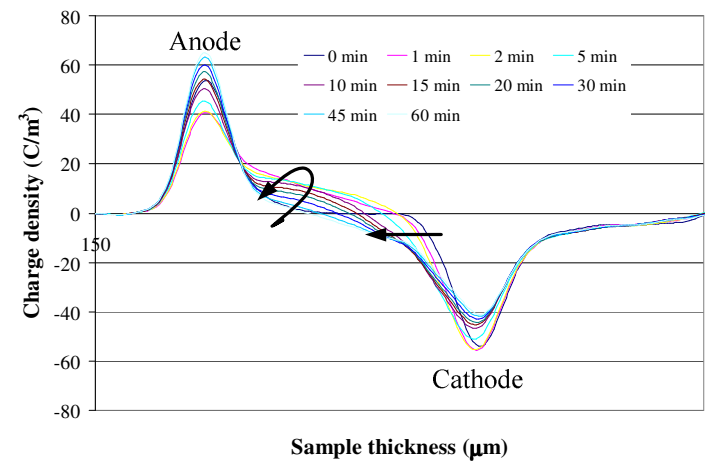

Figure 1 Charge dynamics in control sample at 50 $\mathrm{kV} / \mathrm{mm}$.

It can be seen that charge injection starts immediately when an electric field of $50 \mathrm{kV} / \mathrm{mm}$ is applied. Injection takes place at both electrodes. Initially, positive charge injection is stronger and the injected charges move quickly towards the cathode. This makes the electric field at the cathode increase significantly, leading to more injection from the cathode. Negative charges then spread towards the anode and they gradually dominate charge in the bulk. More negative charge is evident after the removal of the applied voltage at 60 minutes as shown in Fig. 2.

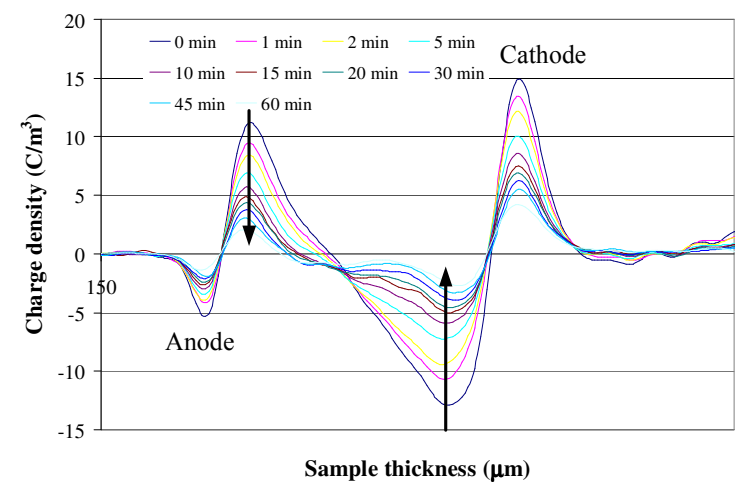

Figure 2 Charge decay dynamics in control sample after the removal of the applied voltage.

Charge dynamics after the removal of the applied voltage is shown in Figure 2. Generally charge decays fairly fast and significant reduction in bulk charge is observed. Another feature is the charge distribution maintains a similar shape. Under the influence of its own field, charge could either leak out from electrodes or recombine. The amount of charge in the bulk can be estimated using the following equation:

$$
Q=\int_{0}^{d}|\rho(x)| \cdot S \cdot d x
$$

where $\rho(x)$ is charge density, S the electrode area and $d$ the thickness of the sample. Both positive and negative charge can also be calculated. Charge decays for total, positive and negative charges in the bulk are shown in Figure 3. As expected from Figure 2, charge decays fairly fast, indicating the presence of shallow traps.

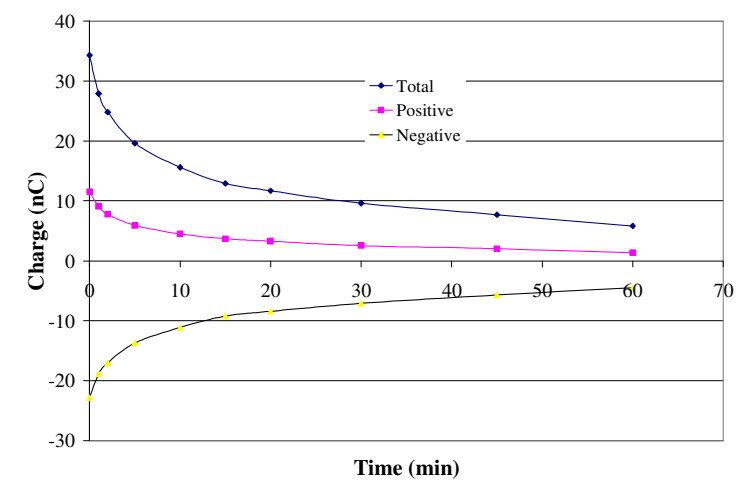

Figure 3 Charge decay in control sample after being stressed at $50 \mathrm{kV} / \mathrm{mm}$ for 60 minutes.

\section{Irradiation in Air}

Figure 4 shows the charge distribution at $50 \mathrm{kV} / \mathrm{mm}$ in a sample irradiated to $5 \mathrm{kGy}$.

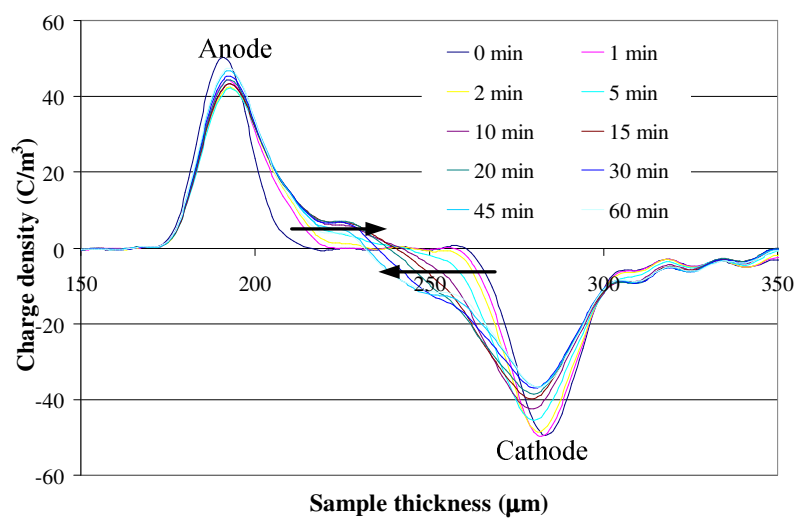

Figure 4 Charge dynamics in $5 \mathrm{kGy}$ irradiated sample at $50 \mathrm{kV} / \mathrm{mm}$.

Charge dynamics are slightly different from that in control sample. Charge injection takes places at both electrodes but there is no significant reduction in positive charge. Over long period negative charges still dominate. This can be seen when the applied electric field is removed as shown in Figure 5.

Comparing Figure 5 to Figure 2, there are two noticeable features. The amount of positive charge in irradiated sample is more and there is a clear separation between positive and negative charge. Again, the rate of reduction in the amount of charge is fast.

Figure 6 shows charge dynamics in 100 kGy irradiated sample at $50 \mathrm{kV} / \mathrm{mm}$. This is a quite different situation compared to what happens at low dose. Although the initial injection at both electrodes is still true, the ultimatum charge distribution is governed by positive 
charge. The reason for this is that the density of ion pairs produced by gamma irradiation increases with increasing radiation dose [9] and therefore they are far more prevalent at $100 \mathrm{kGy}$ than at $5 \mathrm{kGy}$.

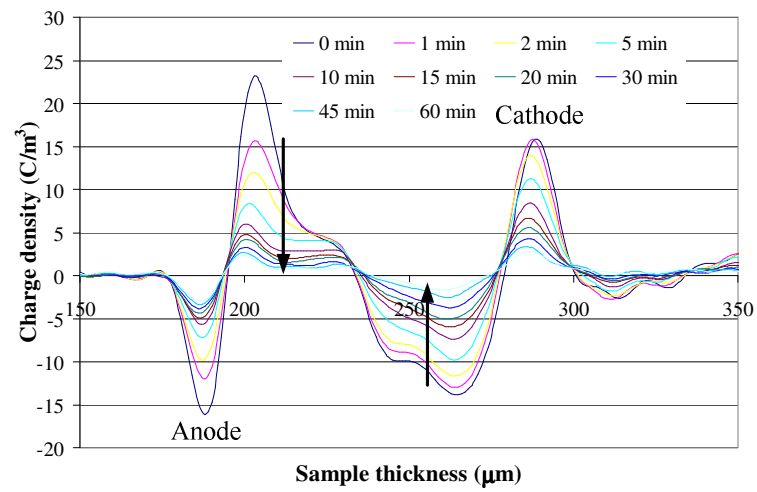

Figure 5 Charge dynamics in 5 kGy irradiated sample after the removal of the applied voltage.

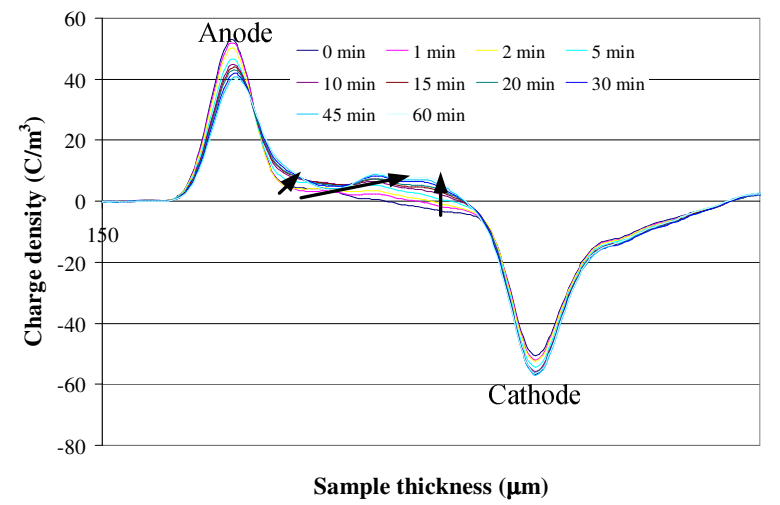

Figure 6 Charge dynamics in $100 \mathrm{kGy}$ irradiated sample at $50 \mathrm{kV} / \mathrm{mm}$

Charge decay dynamics in $100 \mathrm{kGy}$ irradiated sample are shown in Figure 7. In addition to positive charge adjacent to the anode present in all cases, there is a considerable amount of positive charge adjacent to the cathode. In considering this situation it must be borne in mind that while the majority of electrons from ion-pair production are geminate, in that they remain within the sphere of the influence of their parent ions, some are non-geminate and are free to move around in the material by means of thermal excitation and/or the action of an electric stress. In these circumstances trapping and detrapping will play an important role in the population dynamics. In the particular case of the results of Figure 7 it would appear that electrons have been swept by the electric field towards the anode and left the more massive and less mobile positive ions at the cathode. There is a small amount negative charge present between the positive charge regions and they are fairly stable.

The less mobile charge should be reflected in charge decay with time as shown in Figure 8. A slow decay in positive charge is observed. It is believed that electrons are injected from the electrodes to neutralize positive ions.

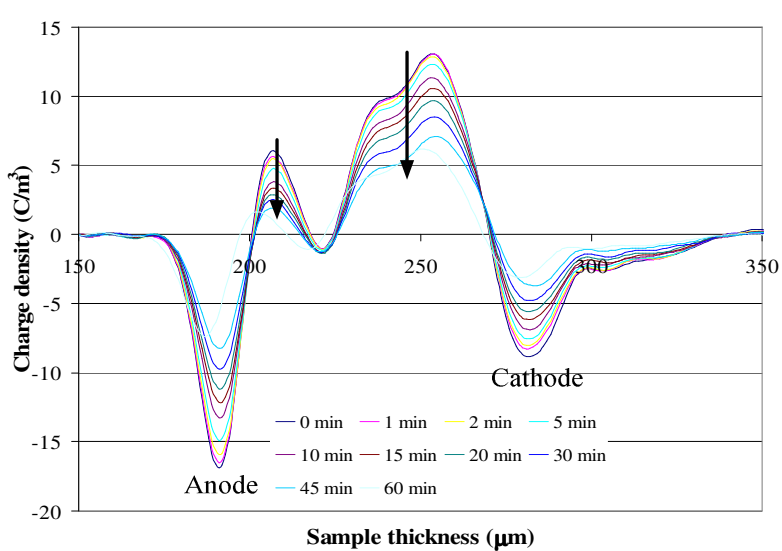

Figure7 Charge decay dynamics in 100 kGy irradiated sample after the removal of the applied voltage.

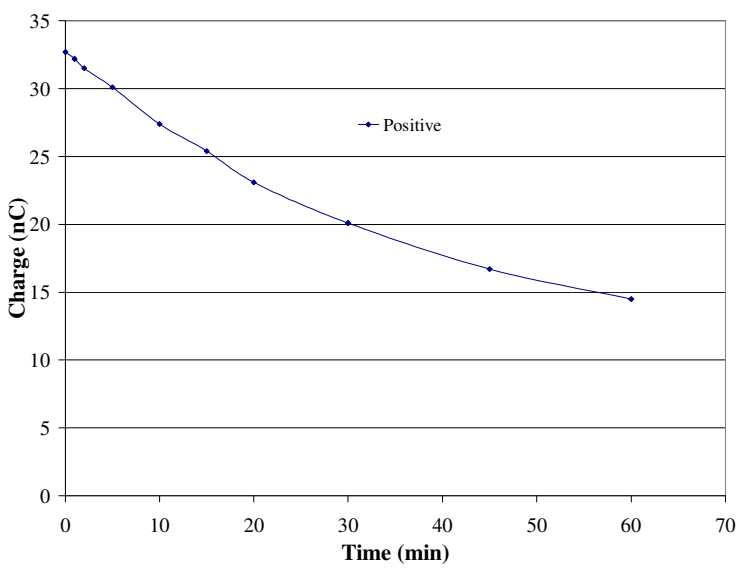

Figure 8 Charge decay in 100 kGy irradiated sample after being stressed at $50 \mathrm{kV} / \mathrm{mm}$ for 60 minutes.

Ionisation of the material also gives rise to radicals and to the subsequent alteration of the structure of the material via chemical radical reactions and the mechanisms of cross-linking, chain scission, gas production and oxidation cited earlier. Thus as well as releasing charge within the material, radiation causes it to change from its original state. This happens not only as irradiation takes place, but also afterwards as oxygen, for example, diffuses into the bulk and produces oxidation products such as ketones, alcohols and hydroperoxides among many others, depending on the material in question. In the presence of oxygen, one oxidation product that is readily observable with irradiated LDPE is the carbonyl group $(\mathrm{C}=\mathrm{O})$. It is well established that the concentration of carbonyl groups increases with radiation dose and that the carbonyl groups generate a shallow trap that readily assists charge transport [10].

\section{Irradiation in Vacuum}

Charge distribution of space charge in a sample exposed to $100 \mathrm{kGy}$ in vacuum is shown in Figure 9 for a stress of $10 \mathrm{kV} / \mathrm{mm}$. Due to length limit, here are only presented charge decay dynamics. 


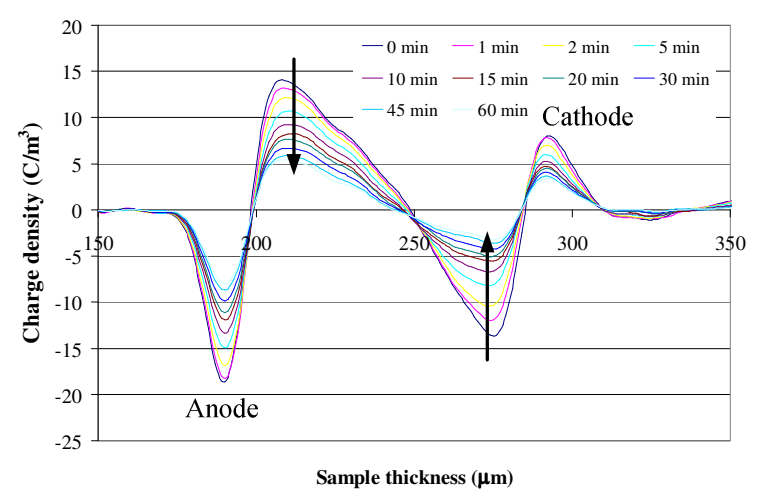

Figure 9 Charge decay dynamics in 100 kGy irradiated sample in vacuum.

Charge distribution after the removal of the applied voltage is similar to that in control sample. However, the positive charge is slightly more than negative charge in irradiated sample. The charge decay rate is much slower than that in control sample. More importantly, charge distribution is completely different from 100k kGy irradiated in air.

In the absence of oxygen, LDPE undergoes predominantly cross-linking under irradiation. The results suggest that the cross-linking by irradiation have minor effect on the charge formation in LDPE but major effect on charge decay.

\section{Irradiation in Nitrogen}

Figure 10 shows the charge distribution in the sample exposed to $100 \mathrm{kGy}$ in nitrogen gas at a stress of 50 $\mathrm{kV} / \mathrm{mm}$. Only negative charges were observed at the end of stressing period. As negative charge peak is adjacent to the cathode, negative charges are believed to be injected from the cathode. Charge decay rate is slow compared to the control sample. The difference in charge distribution among vacuum and nitrogen gas may indicate different chemical changes induced by irradiation.

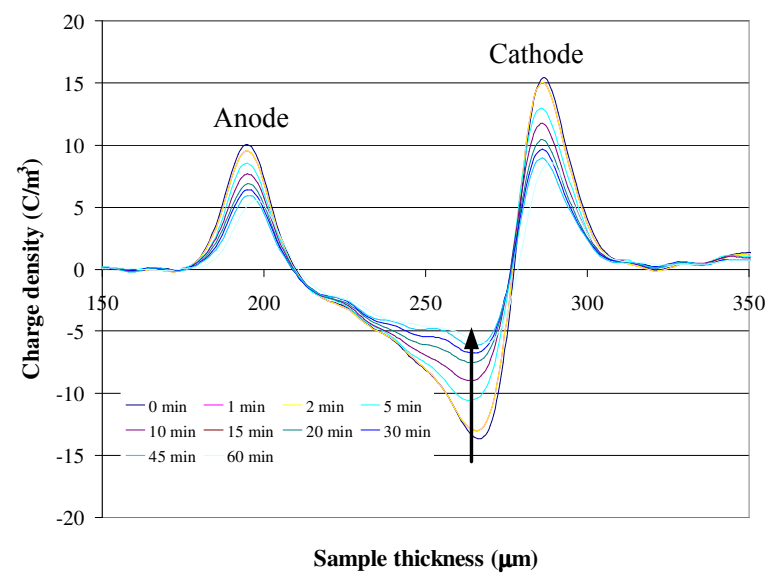

Figure 10 Charge decay dynamics in 100 kGy irradiated sample in nitrogen gas.

\section{CONCLUSIONS}

There are marked differences between the space charge characteristics of material irradiated in air in comparison with LDPE irradiated in either vacuum or oxygen-free nitrogen gas. It is evident that the presence of oxygen during irradiation is essential for a significant amount of space charge to form. The amount of positive charge increases with the radiation dose.

The manner in which space charge distributions vary with dose and irradiation environments is clearly a function of the structural changes taking place within the LDPE.

\section{REFERENCES:}

[1] A. Charlesby, "Atomic Radiation and Polymers", Pergamon, New York, 1960.

[2] J. F. Kircher and R. E Bowman, "Effects of Radiation on Materials and Components", Reinhold, New York, 1964.

[3] J. N. Anno, "Notes on Radiation Effects on Materials", Hemisphere, Washington, 1984.

[4] G. M. Sessler, "Charge dynamics in irradiated polymers", IEEE Trans. Electr. Insul., Vol. 27, pp 961-973, 1992.

[5] G. Chen, R. A. Fouracre, H. M. Banford and D. J. Tedford, "The effects of gamma-irradiation on thermally stimulated discharge current spectra in low-density polyethylene", Radiat. Phys. Chem., Vol. 37, pp 523-530, 1991.

[6] H. M. Banford, R. A. Fouracre, G. Chen and D. J. Tedford, "Electrical conduction in irradiated low-density polyethylene", Radiat. Phys. Chem., Vol. 40, pp 401-410, 1992.

[7] H. M. Banford, R. A. Fouracre, G. Chen and D. J. Tedford, "Transient current pulses in gamma-irradiated polyethylene", Radiat. Phys. Chem., Vol. 37, pp 605-609, 1991.

[8] Y. Li, M. Yasuda and T. Takada, "Pulsed electro-acoustic method for measurement of charge accumulation in solid dielectrics", IEEE Trans. Dielectrics EI, Vol. 1, pp 188-195, 1994.

[9] R. M. Keyser, K. Tsuji and F. Williams, "The Radiation Chemistry of Macromolecules (ed. by M. Dole,)" Vol. 1, Academic, New York, 1972 pp 145-191.

[10] Y. Takai, K. Mori, T. Mizutani and M. Ieda, "Investigation of traps in gamma-irradiated polyethylene by photostimulated detrapping current analysis" Jpn. J. Appl. Phys. Vol. 15, pp 2341-2347, 1976. 